\title{
Halo Substructure in the SDSS-Gaia Catalogue: Streams and Clumps
}

\author{
G. C. Myeong $^{1 \star}{ }^{\star}$, N. W. Evans ${ }^{1}$, V. Belokurov ${ }^{1}$, N.C. Amorisco ${ }^{2,3}$ \& S.E. Koposov ${ }^{1,4}$ \\ ${ }^{1}$ Institute of Astronomy, University of Cambridge, Madingley Road, Cambridge CB3 OHA \\ ${ }^{2}$ Max Planck Institute for Astrophysics, Karl Schwarzschild Strasse 1, D-85740 Garching, Germany \\ ${ }^{3}$ Institute for Theory and Computation, Harvard Smithsonian Center for Astrophysics, 60 Garden Street, Cambridge, MA02138, USA \\ ${ }^{4}$ McWilliams Center for Cosmology, Department of Physics, Carnegie Mellon University, 5000 Forbes Avenue, Pittsburgh, PA 15213, USA
}

version 13 December 2017

\begin{abstract}
We use the SDSS-Gaia Catalogue to identify six new pieces of halo substructure. SDSS-Gaia is an astrometric catalogue that exploits SDSS data release 9 to provide first epoch photometry for objects in the Gaia source catalogue. We use a version of the catalogue containing 245316 stars with all phase space coordinates within a heliocentric distance of $\sim 10 \mathrm{kpc}$. We devise a method to assess the significance of halo substructures based on their clustering in velocity space. The two most substantial structures are multiple wraps of a stream which has undergone considerable phase mixing (S1, with 94 members) and a kinematically cold stream (S2, with 61 members). The member stars of $\mathrm{S} 1$ have a median position of $(X, Y, Z)=$ $(8.12,-0.22,2.75) \mathrm{kpc}$ and a median metallicity of $[\mathrm{Fe} / \mathrm{H}]=-1.78$. The stars of $\mathrm{S} 2$ have median coordinates $(X, Y, Z)=(8.66,0.30,0.77) \mathrm{kpc}$ and a median metallicity of $[\mathrm{Fe} / \mathrm{H}]=-1.91$. They lie in velocity space close to some of the stars in the stream reported by Helmi et al. (1999). By modelling, we estimate that both structures had progenitors with virial masses $\approx 10^{10} M_{\odot}$ and infall times $\gtrsim 9 \mathrm{Gyr}$ ago. Using abundance matching, these correspond to stellar masses between $10^{6}$ and $10^{7} M_{\odot}$. These are somewhat larger than the masses inferred through the mass-metallicity relation by factors of 5 to 15 . Additionally, we identify two further substructures (S3 and S4 with 55 and 40 members) and two clusters or moving groups (C1 and $\mathrm{C} 2$ with 24 and 12) members. In all 6 cases, clustering in kinematics is found to correspond to clustering in both configuration space and metallicity, adding credence to the reliability of our detections.
\end{abstract}

Key words: galaxies: kinematics and dynamics - galaxies: structure

\section{INTRODUCTION}

Lord Rutherford briskly asserted "All Science is either Physics or Stamp Collecting". The study of the stellar halo of the Milky Way has seen much philately over the last decade with the discovery of abundant streams and substructure (e.g., Belokurov et al. 2006; Grillmair 2009; Newberg \& Carlin 2015). These have usually been identified as overdensities from resolved star maps. Substructures remain kinematically cold and identifiable in phase space long after they have ceased to be recognizable in star counts against the stellar background of the Galaxy. In principle, searches in velocity space or in phase space are much more powerful than direct searches in configuration space. There are believed to be hundreds of accreted dwarf galaxies and globular clusters in the halo of the Milky Way which could be found through searches in velocity space.

In practice, kinematic data has been so fragmentary to date

^ E-mail: gm564,nwe,vasilyast.cam.ac.uk,

koposov@cmu.edu, nicola.amorisco@cfa.harvard.edu that such substructure searches have been difficult to perform. There have been some successes, such as the group of 8 stars in the Hipparcos data clumped in metallicity and phase space found by Helmi et al. (1999) or the discrete kinematic overdensities in Sloan Digital Sky Survey (SDSS) Stripe 82 identified by Smith et al. (2009). Nonetheless, given the ostensible power of the method, results have been meagre.

The advent of data from the Gaia satellite (Gaia Collaboration et al. 2016a) is a pivotal moment for identifying the hundreds of partially mixed phase space structures that numerical simulations suggest should be present in the halo. Many of these have dissolved sufficiently to fall below the surface brightness threshold of current imaging surveys, and thus will remain unnoticed without kinematic data from Gaia.

The first Gaia data release provided TGAS, or the Tycho-Gaia Astrometric Solution, which used the earlier Tycho catalogue as the first epoch for the astrometric solution (Gaia Collaboration et al. 2016b). TGAS gives the proper motions and parallaxes of just over 2 million stars. Subsets of these stars are in ongoing radial 
velocity surveys such as LAMOST, RAVE or RAVE-on (Luo et al. 2015; Casey et al. 2017; Kunder et al. 2017). Already, claims of a coherently moving feature in velocity space (Myeong et al. 2017) as well as over-densities in "integrals of motion space" (Helmi et al. 2017) have been made.

Cross-matches between TGAS and radial velocity surveys produce catalogues of $\sim 250000$ stars. These are primarily local samples, dominated by denizens of the local disk within $1 \mathrm{kpc}$. It would be advantageous to use a much larger and deeper sample of stars with full phase space information. Along with TGAS, Gaia data release 1 also comprised the Gaia source photometric catalogue, which provides the locations of $\sim 10^{9}$ sources. Koposov (2017, in prep.) recalibrated the SDSS astrometric solution and then obtained proper motions from Gaia positions and their recalibarted positions in SDSS. This catalogue is also discussed in some detail in Deason et al. (2017) and de Boer et al. (2018). The individual SDSS-Gaia proper motions have statistical errors typically $\sim 2$ mas $\mathrm{yr}^{-1}$, or $\sim 9.48 D \mathrm{~km} \mathrm{~s}^{-1}$ for a star with heliocentric distance $D \mathrm{kpc}$. As the SDSS data were taken over a significant period of time, the error is primarily controlled by the time baseline. However, there are no systematic effects down to a level of $0.1-0.2$ mas $\mathrm{yr}^{-1}$ in the astrometry with regard to magnitude or colour (see e.g., Figure 2 of Deason et al. 2017), so this makes the SDSS-Gaia catalogue suitable for searching for large-scale velocity signatures corresponding to streams and substructures.

The depth of SDSS-Gaia enables us to search out to heliocentric distances of $\sim 10 \mathrm{kpc}$, which is a substantial advantage over TGAS. Cross-matching SDSS-Gaia with spectroscopic surveys can add radial velocities. Finally, photometric parallaxes for stars such as main-sequence turn-offs (MSTOs) or blue horizontal branch stars (BHBs) gives samples with the full six-dimensional phase space coordinates. Although the SDSS-Gaia catalogue will be superseded in April 2018 by the next Gaia data release, it currently provides the best catalogue in which to search for halo substructure by kinematic means.

The overall aim of this activity is to constrain the fraction of halo stars in clumps and substructures. This is of great interest as it encodes the accretion history of the stellar halo and by extension of the Milky Way itself. Nonetheless, the optimum algorithms for substructure identification, as well as the best methodologies to match detected substructures to disrupting subhalos in numerical simulations, are ripe for exploration with SDSS-Gaia. Ultimately, a better understanding of such algorithms is needed to convert the 'stamp collecting' into astrophysics.

In this spirit, Section 2 introduces a new method to search for substructure in velocity space in the SDSS-Gaia catalogue. The six most significant halo substructures are studied in detail in Section 3. They include a gigantic stream with cold kinematics, two moving groups and three hotter substructures in which the velocity distribution in at least one component is very broad. By matching with substructure in a library of numerical simulations in Section 4 , we argue that hotter substructures probably correspond to multiply-wrapped streams in the later stages of disruption. For the two largest substructures, we provide estimates of the likely mass of the progenitor and infall time. Finally, Section 5 sums up with an eye to possible extensions and elaborations of our new method.

\section{METHOD}

\subsection{Sample}

Our starting sample is the crossmatch between Gaia data release 1 (DR1), the Sloan Digital Sky Survey data release 9 (PhotoObjAll for the photometric and sppParams for the spectroscopic), APOGEE, LAMOST DR2 and RAVE-on (see e.g., Anders et al. 2014; Luo et al. 2015; Casey et al. 2017; Kunder et al. 2017). There are 466414 stars in this sample with five-dimensional phase space information. The sample contains MSTO stars and BHB stars, which can be extracted using methods similar to Sections 3.1 and 3.2 of Williams et al. (2017). The MSTO stars are extracted using the cuts: extinction $\epsilon_{r}<0.5, g, r, i$ magnitudes satisfying $14<g<20,14<r<20,14<i<20,0.2<(g-r)_{0}<0.8$ with surface gravity $3.5<\log g<5.0$ and effective temperature $4500<T_{\text {eff }}<8000$. The BHB stars are chosen from $-0.25<$ $(g-r)_{0}<0.0,0.9<(u-g)_{0}<1.4$ with spectroscopic parameters satisfying $3.0<\log g<3.5$ and $8300<T_{\text {eff }}<9300$. We apply a set of quality cuts to both the photometric and spectroscopic data to remove stars with uncertain measurements as well as stars with a heliocentric radial velocity error $>15 \mathrm{kms}^{-1}$ and a heliocentric distance $>10 \mathrm{kpc}$. The cuts cause the sample to be reduced to 245316 in size with 245078 MSTO stars and 238 BHB stars. The median heliocentric radial velocity error is $2.9 \mathrm{kms}^{-1}$ and the median proper motion error is $17.8 \mathrm{kms}^{-1}$. Parallaxes can be obtained via the formulae in Ivezic et al. (2008) for MSTOs (using spectroscopic metallicities) and in Deason et al. (2011) for BHBs to give full six-dimensional phase space information. For the MSTOs that comprise the bulk of the sample, mean distance error scales linearly with distance and reaches $\sim 1 \mathrm{kpc}$ at a distance of $4.5 \mathrm{kpc}$. The mode of the distance error for the whole MSTO sample is $\sim 0.47$ kpc.

Velocities in the Galactic rest-frame are resolved in the cylindrical polar coordinate system to give $\left(v_{R}, v_{\phi}, v_{z}\right)$. From the histogram in the $\left(v_{\phi},[\mathrm{Fe} / \mathrm{H}]\right)$ plane in Fig. 1 , we see a reasonably clear separation of the halo population from the thin and thick disk populations. We define a polygon (converted from a contour) representing each population, and then calculate the distance of each star from two contours (one representing the halo, the other representing the thin and thick disks). This enables us to classify each star as either halo or disk. For the halo stars, we perform a Gaussian fitting decomposition based on the metallicity, and then subdivide the halo group into the relatively metal-rich, and the relatively metalpoor halo. As the result of the Gaussian decomposition, the division occurs at $[\mathrm{Fe} / \mathrm{H}] \approx-1.65$. Our sample then comprises 181574 disk stars (green), 40293 relatively metal-rich halo stars (blue), and 23449 relatively metal-poor halo stars (red), as shown in Fig. 1.

This subdivision of the stars into disk and halo groups is crude, but we only wish to use it to demonstrate that the sequence from thin and thick disk through metal-rich halo to metal-poor halo is one of increasing substructure. This is evident from Fig. 2 in which the logarithmic contours of the velocity distribution in the $\left(v_{R}, v_{\phi}\right)$ plane moves from smoothness to raggedness with increasing numbers of outliers and subgroups. Some of this effect is statistical in origin as there are between 4 and 8 times fewer stars in the halo populations. However, some prominent pieces of halo substructure can be picked out by eye, and so some of the effect is real. Accordingly, we proceed to develop a systematic way of identifying the substructure. 


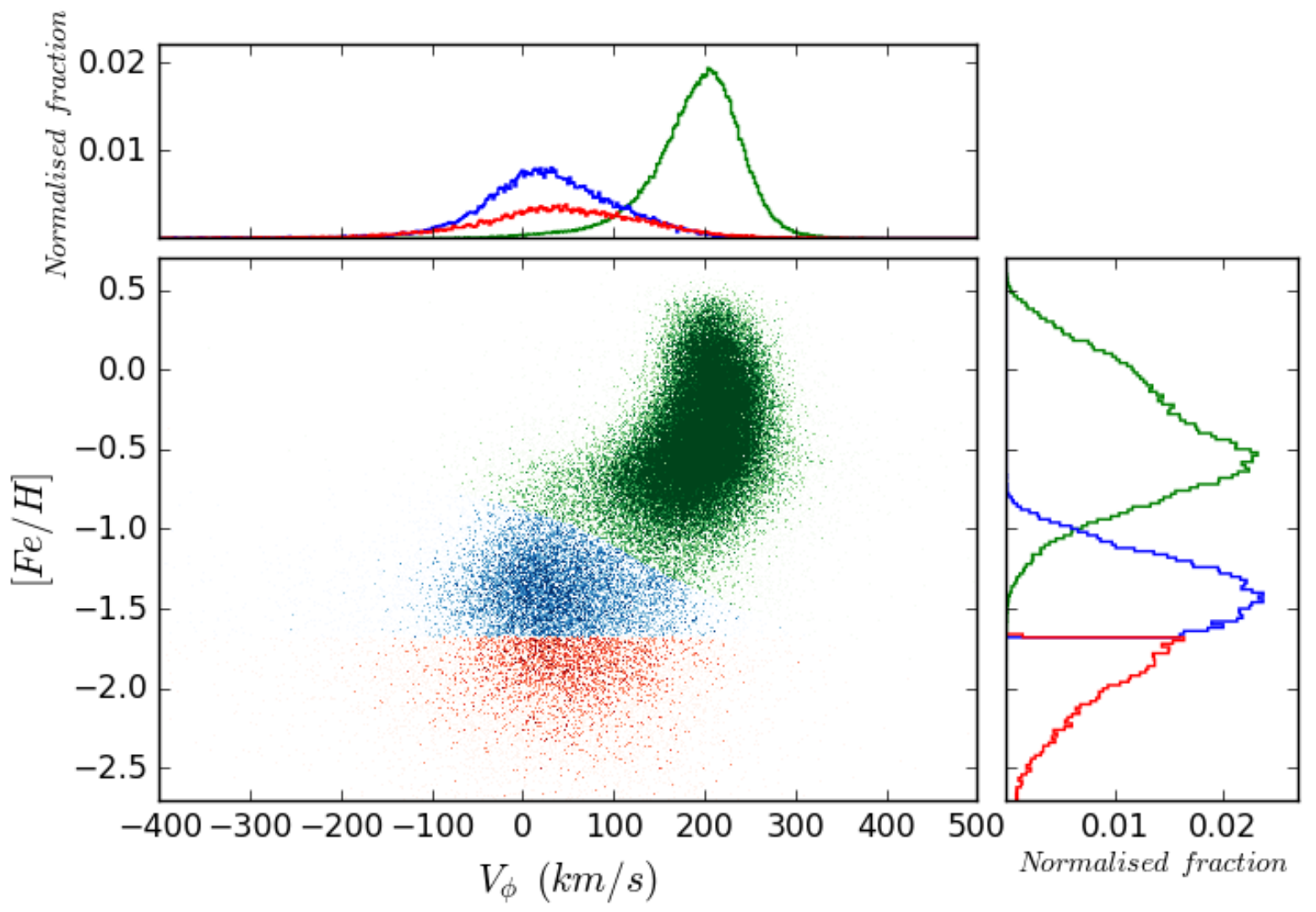

Figure 1. The cleaned sample is shown in the $\left(v_{\phi},[\mathrm{Fe} / \mathrm{H}]\right)$ plane. There is a clear separation of the halo stars from the disk (thin and thick) populations. Green represents the disk, blue the relatively metal-rich halo $([\mathrm{Fe} / \mathrm{H}]>-1.65)$, and red the relatively metal-poor halo $([\mathrm{Fe} / \mathrm{H}]<-1.65)$. For the one dimensional $v_{\phi}$ and $[\mathrm{Fe} / \mathrm{H}]$ distributions, the normalisation is performed separately for the disk, and for the entire halo group, so the sum of the area under the green histogram is unity, as is that for the blue and red combined.

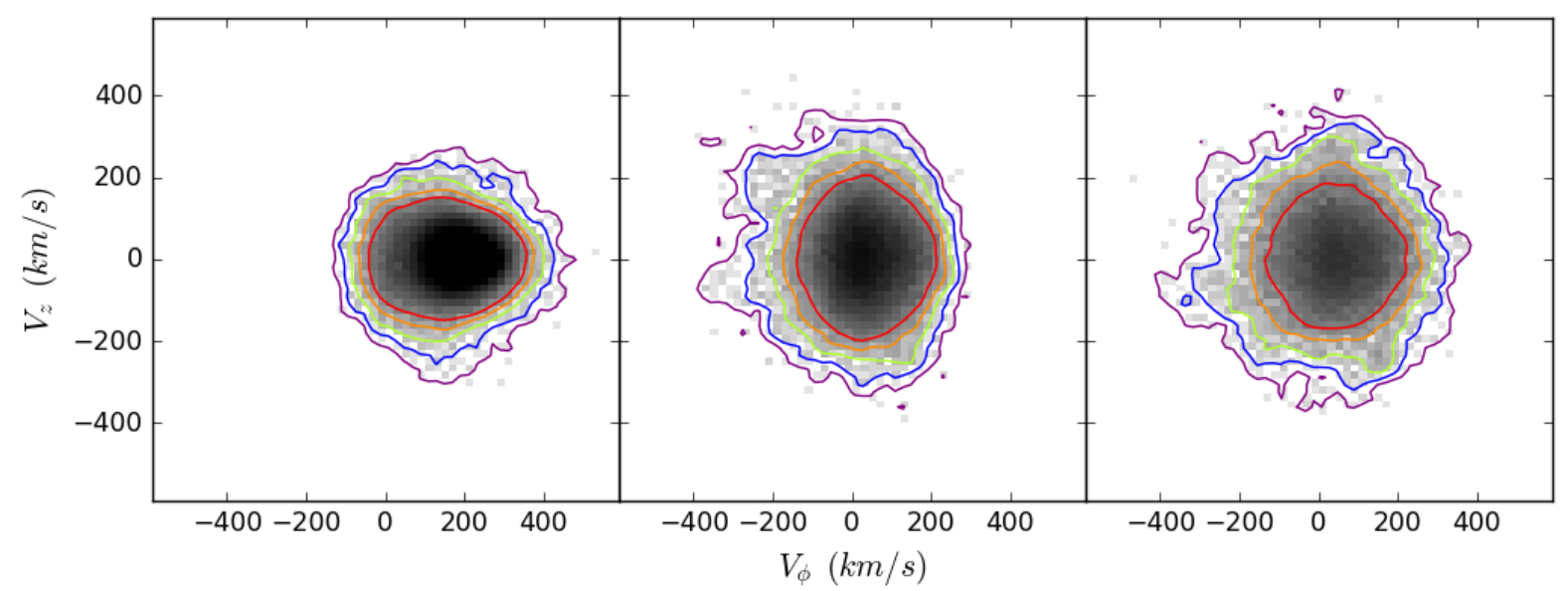

Figure 2. The data are shown in the plane of $\left(v_{\phi}, v_{z}\right)$ for the disk (left), metal-rich halo (middle) and metal-poor halo (right). The contours levels are logarithmic. We can see visible substructure evident in the metal-rich $([\mathrm{Fe} / \mathrm{H}]>-1.65)$ and metal-poor $([\mathrm{Fe} / \mathrm{H}]<-1.65)$ halo groups. It is apparent that the sequence from disk to metal-rich halo to metal-poor halo is one of increasing lumpiness and substructure. The pixel size is $20 \mathrm{kms}^{-1}$ on each side. The outermost contour is 2 stars per pixel

$$
\text { , and the contours increase by a factor of } 10^{0.35} \approx 2.24 \text { on moving inwards. }
$$



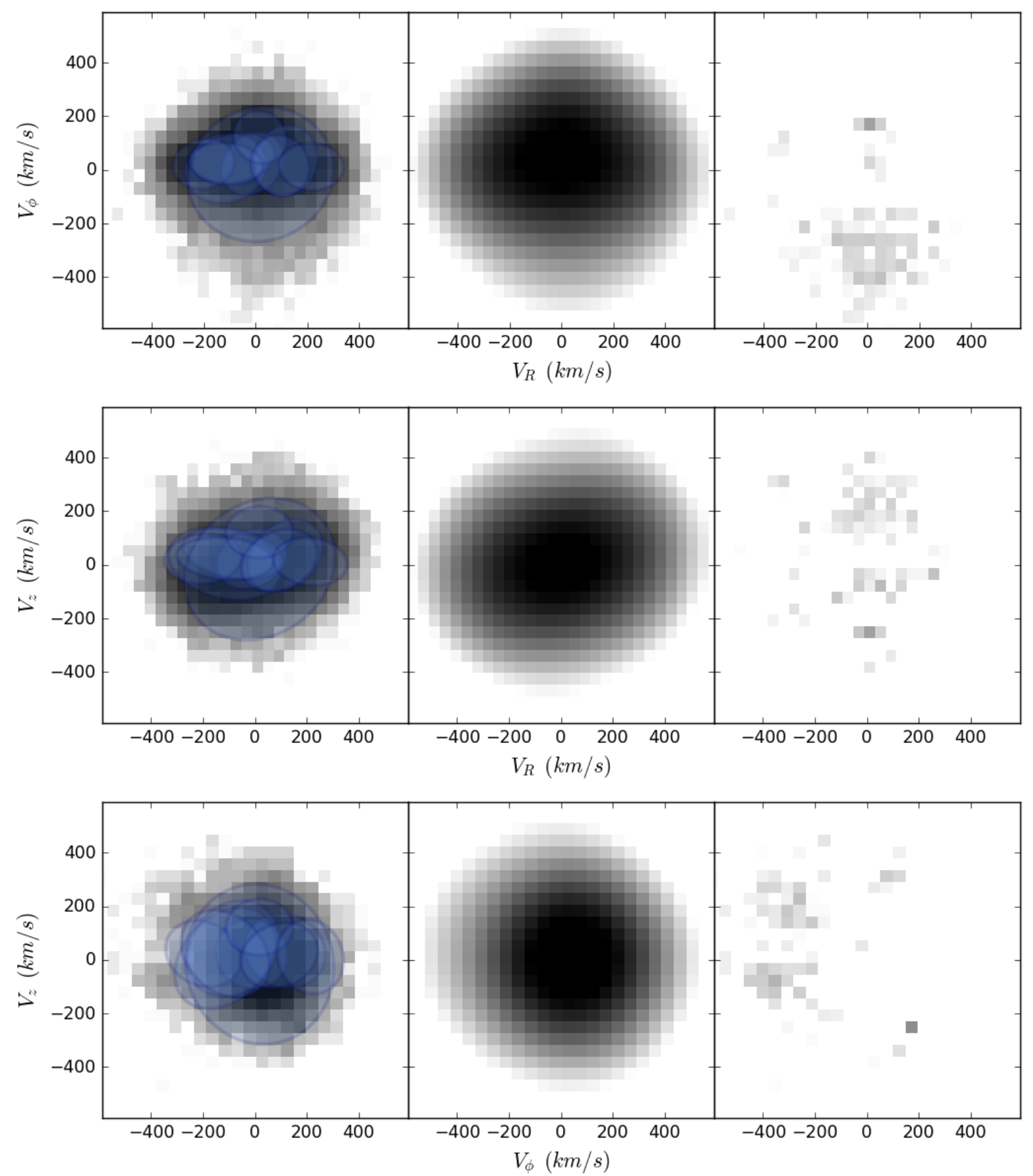

Figure 3. For the entire halo sample, we show from left to right the data, the smooth Gaussian Mixture model, and the residuals. Superposed on the data are blue ellipses representing the Gaussians with orientation and sizes scaled according to their principal axes. The rows show the principal planes in velocity space $\left(v_{R}, v_{\phi}\right),\left(v_{R}, v_{z}\right)$ and $\left(v_{\phi}, v_{z}\right)$ respectively. Although the Gaussian mixture model is a good representation of the halo, substructure is already apparent in the plots in the rightmost column. The residuals demonstrate the locations of the main pieces of substructure, as well as highlighting the lumpy nature of the distribution.

\subsection{Detection}

Henceforth, we use the entire halo sample (the blue and red distributions in Fig. 1). We first develop a smooth underlying background model, which is then used as the global density estimator against which substructure is identified. Using Galactocentric velocities resolved with respect to cylindrical polar coordi- nates $\left(v_{R}, v_{\phi}, v_{z}\right)$, we fit a basic Gaussian Mixture model from the Scikit-learn (Pedregosa et al. 2011) python software package ${ }^{1}$. Note that if we use too many Gaussian components, some of the actual signals from genuine substructures get diluted by some of the

\footnotetext{
${ }^{1} \mathrm{http}: / /$ scikit-learn.org
} 

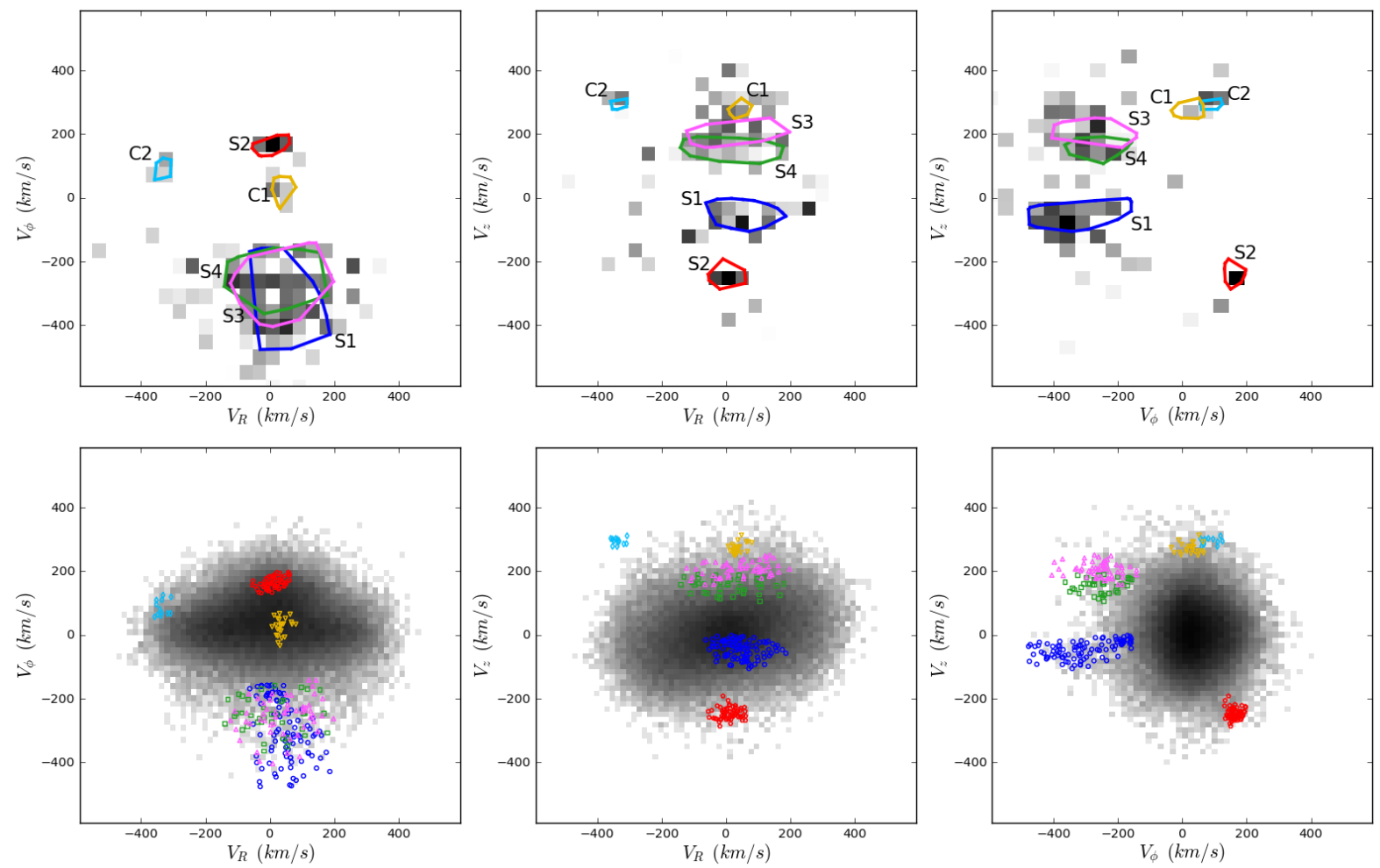

Figure 4. The velocity distributions of the full halo sample (bottom row) and the residuals (top row) are shown in the three principal planes in velocity space, $\left(v_{R}, v_{\phi}\right),\left(v_{R}, v_{z}\right)$ and $\left(v_{\phi}, v_{z}\right)$. Stars belonging to the two most prominent substructures are shown as blue circles and red pentagons (S1 and S2). Also shown are two smaller substructures as upward-pointing magenta triangles and green squares (S3 and S4), and two moving clumps as downward-pointing brown triangles and pale blue diamonds (C1 and $\mathrm{C} 2)$.

fitted Gaussians. To avoid such dilution, we decide to use considerably less Gaussian components than the estimate of the number of components obtained from minimization of the Akaike Information Criterion or AIC test (56 components). We ensure that each of the fitted Gaussians has a width wider than $150 \mathrm{kms}^{-1}$ on each axis to avoid including small scale features in our velocity distribution model. We find that 10 Gaussians provide a reasonable description of the velocity space for the halo stars, as shown in Fig. 3. The data, together with the superposed Gaussians are shown in the left panels, whilst the smooth model and residuals are shown in the middle and right. It is evident that there is substructure, and much of it corresponds to prominent clumps in Fig 2.

Next, we look for significant overdensities over the Gaussian Mixture model. We measure the local density of each star in our data, and compare this to the density value predicted by the smooth model. We do this by carrying out a $k$-nearest neighbours search with $k=5$ (or 6 including the star itself). Using Scikit-learn, we obtain the radius $r_{5}$ required to encounter the $k=5$ nearest neighbours and hence an estimate of the local density. The probability of the star's location in the 3-dimensional velocity space is predicted by the Gaussian Mixture model. We multiply this by the sample size and the volume of the sphere with radius $r_{5}$ to give the expected number. We assume Poisson sampling and from the expected number of stars in the sphere, we compute the tail probability of having 6 stars (5 neighbours and the star itself) in the sphere given this distribution. We then convert the tail probability to the number of sigma.

We use any stars with significance $>4$ as the "seeds" for finding an overdensity in our 3-dimensional velocity space. First, we classify these seeds by the Friends-of-Friends method - that is, any seeds that are close to each other $\left(<30 \mathrm{kms}^{-1}\right.$ radius sphere $)$ are considered as the same group. For each seed, we then take all stars within a spherical volume of radius $35 \mathrm{kms}^{-1}$ around the seed. During this process, we discard any seeds and corresponding stars if there exist less than 5 stars within this spherical volume. We classify the stars around the seeds by using the Nearest Neighbours Classification from Scikit-learn. This stage is necessary because there are cases in which a star is picked up by more than one seed. So we train the classifier using the classified seeds, and then perform a distance-weighted $k$ neighbours classification $(k=3)$ for the stars around the seeds. The weight here is the inverse of the distance. This gives us a list of candidates.

Now, we find the centre of each group in our 3-dimensional space. The measured number of group members is the number of stars in the ellipsoid in velocity space occupied by the group. This ellipsoid has a volume $4 \pi a b c / 3$, where $(a, b, c)$ is the extent of the group in each axis. The expected number of field stars in the volume ellipsoid is then the probability predicted by the Gaussian Mixture Model at the central location multiplied by the data size and by the volume. The Poisson uncertainty is the square root of the expected number. This provides us with a crude measure of the significance of each substructure.

We will provide the list of substructures elsewhere, but here we describe the six most significant pieces of halo substructure, which is $\sim 20$ per cent of the detected potential candidates with $\sigma>4$. They are labelled $\mathrm{S}$ for stream or shell-like substructures and $\mathrm{C}$ for clusters or moving groups. The locations of the stars in velocity space belonging to the substructures are shown in Fig. 4. Note 
S1
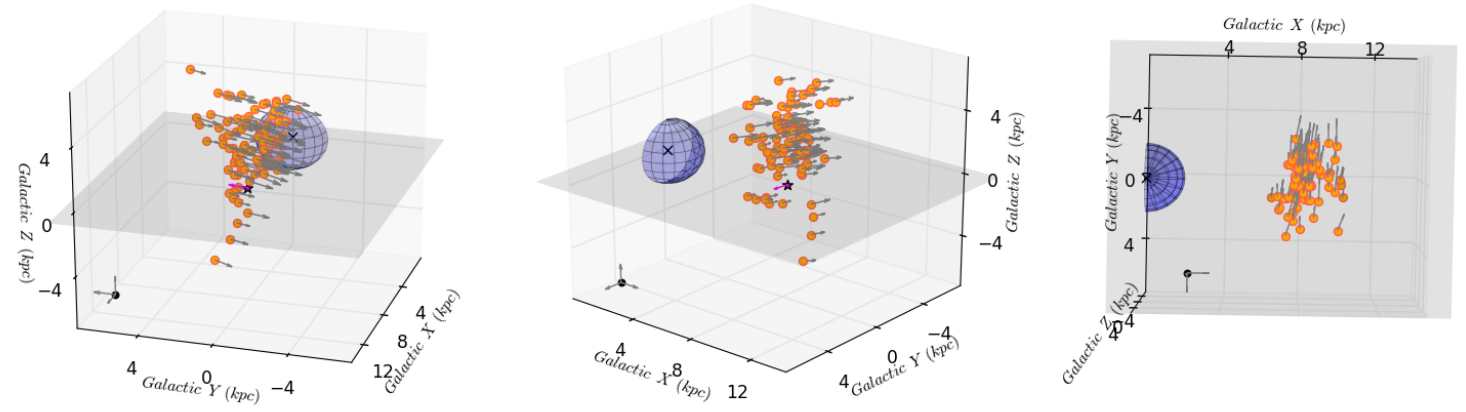

S3
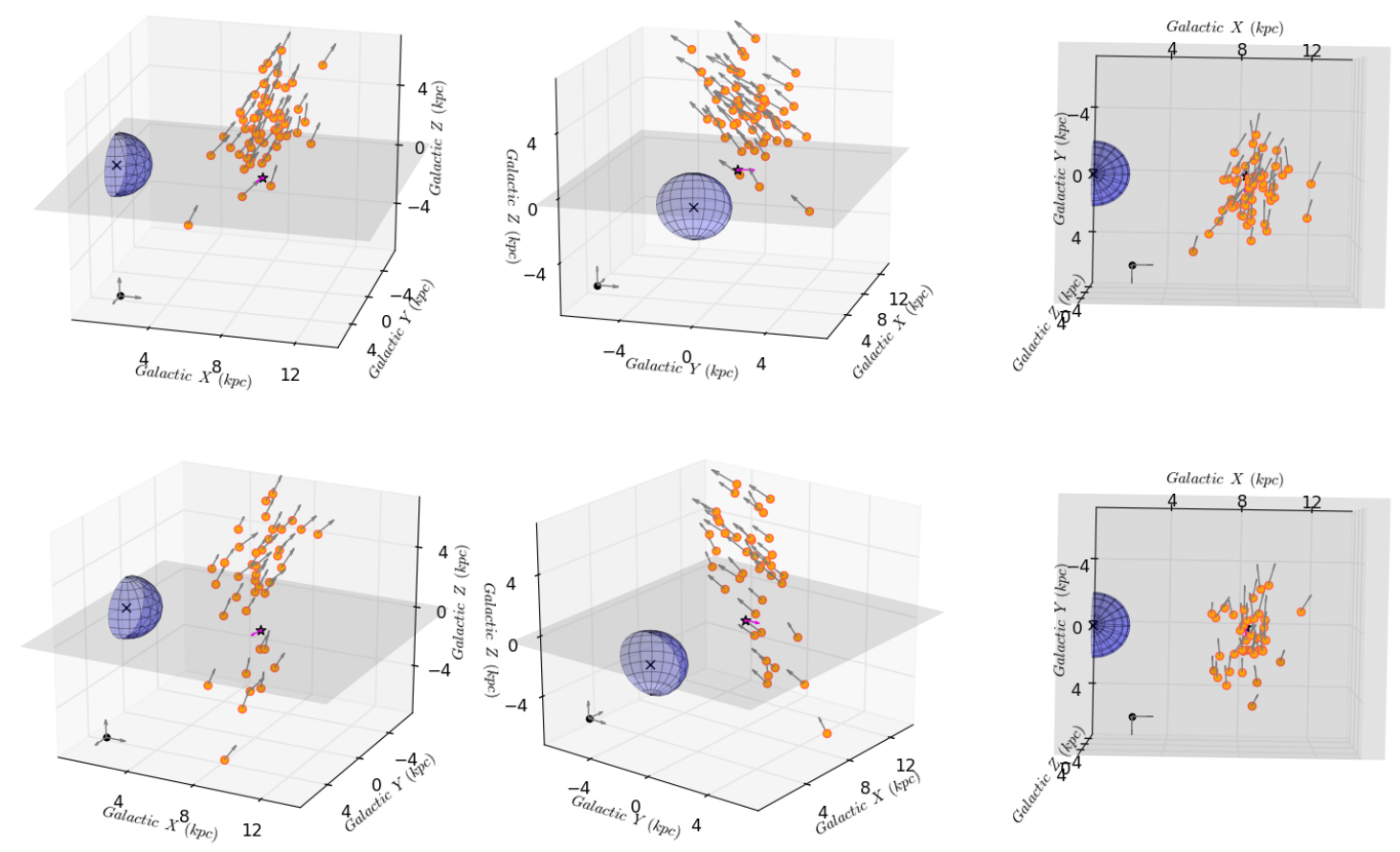

Figure 5. The properties of stars belonging to the three substructures S1, S3 and S4. We have grouped them together because of the morphological similarity. The left and middle panes show two views of the substructure with the intention of depicting the overall shape. The right panel is a projection of the substructure onto the Galactic plane. The arrows show the total velocity in the Galactic rest-frame. The Sun is marked as a star at the centre, whilst the Sun's motion is marked by an arrow in magenta. A sphere of radius $2 \mathrm{kpc}$ (which is a crude representation of the Galactic bulge), as well as a grey sheet representing the Galactic plane, are shown to give a sense of the scale and position of the substructure in relation to the familiar Galactic landscape. A triad of velocity vectors of scale $300 \mathrm{kms}^{-1}$ is shown in the bottom left corner.

that, as the stars lie within the SDSS footprint, proper motions contribute mainly to the radial $v_{R}$ and azimuthal $v_{z}$ components, whilst the line of sight velocities contribute mainly to $v_{z}$. This causes kinematic features to appear colder in $v_{z}$ than in the other two directions which are more affected by errors. The two largest substructures in terms of the number of member stars are S1, coloured blue, with 94 identified members $(\sigma=8.94)$ and $\mathrm{S} 2$, coloured red, with 61 members $(\sigma=8.95)$. Just behind them in terms of the number of member stars are: S3, coloured magenta, with 55 members $(\sigma=8.41)$ and $\mathbf{S} 4$, coloured green, with $40(\sigma=8.49)$ members. There are also two clumps or moving groups: $\mathrm{C} 1$, coloured brown, with 24 members $(\sigma=8.46)$ and $\mathrm{C} 2$, coloured pale blue, with 12 members $(\sigma=18.66)$. Table 1 provides the median, mean absolute deviation and dispersion for kinematical and spectroscopic quantities of the substructures. A list of stars in the substructures is available electronically from the authors.

\section{CANDIDATES}

\subsection{The Hotter Substructures: S1, S3 and S4}

Fig. 5 shows the discovery panels for the three hotter substructures. For each, we provide two views of the morphology in the left and middle panels, as well as a projection onto the Galactic plane on the right. The metallicity distribution function of each substructure is compared against that of the full halo sample in Fig. 7.

$\mathrm{S} 1$ is a large piece of halo substructure, containing $94 \mathrm{mem}-$ ber stars. The members correspond to an obvious narrow tail-like overdensity in the $\left(v_{\phi}, v_{z}\right)$ velocity distribution in Figs 2 or 4 , visible by eye. The medians of the positions of the stars provide a location of $(X, Y, Z)=(8.1,-0.2,2.8) \mathrm{kpc}$, so the structure lies just beyond the Sun's location. It has a substantial extension in both $Y$ and $Z$ as indicated by the median absolute deviations of $\sim 1 \mathrm{kpc}$, so it is distended vertically and azimuthally. Therefore, the spa- 

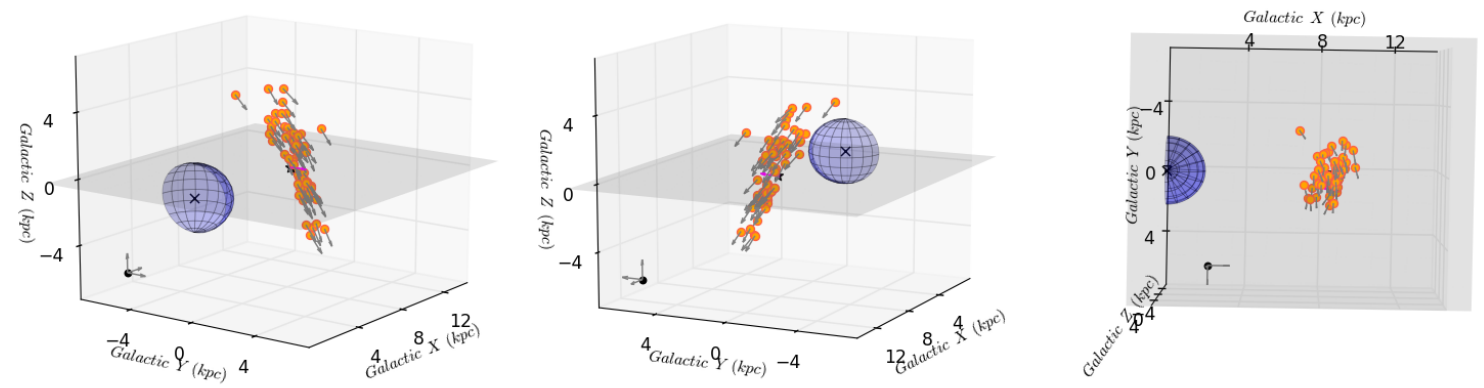

$\mathrm{C} 1$
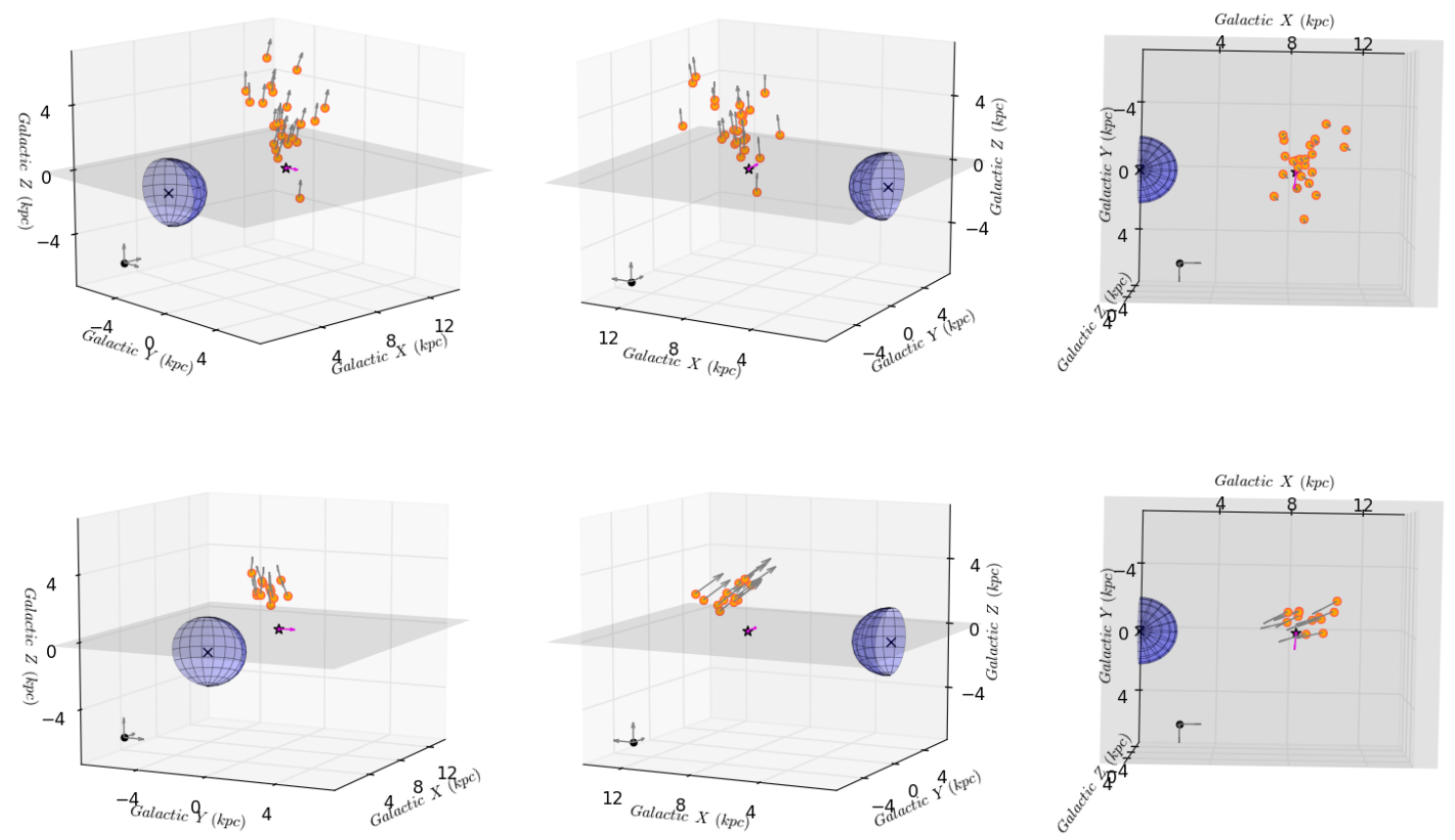

Figure 6. As Fig. 5, but for the stream S2 and the two moving clumps (C1 and C2).

tial configuration is shell-like, pirouetting around the Sun's location. The vertical or $v_{z}$ velocities are tightly constrained around a median of $-42.7 \mathrm{kms}^{-1}$ with a median absolute deviation of 21.3 $\mathrm{kms}^{-1}$. The structure is counter-rotating with a median $v_{\phi}$ of -313.8 $\mathrm{kms}^{-1}$. The median radial velocity $v_{R}$ is $44.8 \mathrm{kms}^{-1}$ with a comparatively large median absolute deviation of $38.4 \mathrm{kms}^{-1}$, mainly caused by the extent of the structure. It is natural to inquire whether this is a diminutive analogue of the shell-like features seen in elliptical galaxies (Hernquist \& Quinn 1987) or in the Milky Way halo (Rocha-Pinto et al. 2004; Belokurov et al. 2007). However, shells are known to be associated with radial infall of galaxies or clusters (e.g., Amorisco 2015; Hendel \& Johnston 2015; Pop et al. 2017), whereas the strongly counter-rotating nature of the substructure indicates that the progenitor orbit has high angular momentum. We will elaborate on the true nature of this structure in the next Section.

The detection algorithm used to identify substructures is based on kinematics alone. However, in all our presented substructures, it is possible to identify clumpiness in configuration space and in chemical properties. Fig. 7 shows the metallicities of the $S 1$ stars in red are much spikier than the halo metallicity distribution function in green. They have a median metallicity of -1.78 with a narrow median absolute deviation of 0.19 , making this a convincing detection.

S3 and S4 share some similarities with S1 in that the radial and azimuthal velocity distributions are broad, but the vertical velocity distribution is narrower, suggesting a highly inclined orbital plane. S3 and S4 are more obviously stream-like, as the stars are moving along the extent of the structure, whereas S1 moves almost perpendicularly. All three substructures are on retrograde orbits. They all lie just beyond the Solar position, though the preponderance of substructure here is a selection effect of the SDSS-Gaia catalogue. The stars belonging to both $\mathrm{S} 3$ and $\mathrm{S} 4$ are tightly clustered in metallicity with median values of -1.34 and -1.70 respectively. Although S3 and S4 occupy similar region in the 3-dimensional velocity space, they show clear deviation in their metallicity distribution as well as in their $v_{z}$ distribution which suggest they are separate substructures. This has been further checked by the Gaussian fitting decomposition on the 4-dimensional space (3-dimensional velocity components and the metallicity) which shows the separation between 

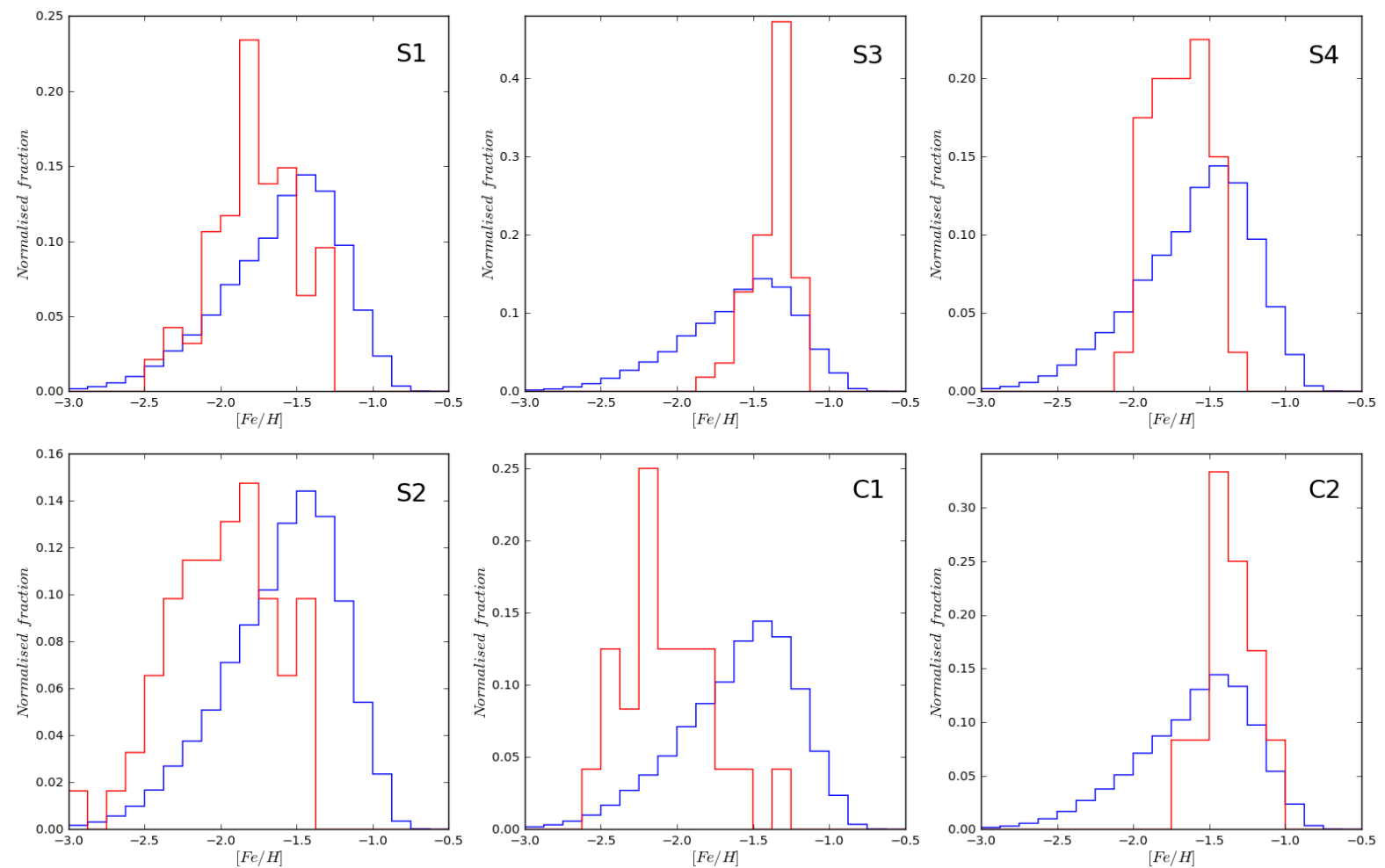

Figure 7. The metallicity distribution function for the six substructures is shown in red, whilst the blue is the entire halo sample for comparison. Note that the substructures are narrower in metallicity than the entire halo, which is consistent with expectations.

\begin{tabular}{|c|c|c|c|c|c|c|c|c|c|}
\hline Name & & {$[\mathrm{Fe} / \mathrm{H}]$} & $\log g$ & $\begin{array}{l}T_{\text {eff }} \\
(\mathrm{K})\end{array}$ & $\begin{array}{c}(X, Y, Z) \\
(\mathrm{kpc})\end{array}$ & $\begin{array}{c}\left(v_{R}, v_{\phi}, v_{z}\right) \\
\left(\mathrm{kms}^{-1}\right)\end{array}$ & $\begin{array}{c}(U, V, W) \\
\left(\mathrm{kms}^{-1}\right)\end{array}$ & $\begin{array}{c}\mathrm{KE} \\
\left(\mathrm{km}^{2} \mathrm{~s}^{-2}\right)\end{array}$ & $\begin{array}{c}\mathrm{L} \\
\left(\mathrm{kpc} \mathrm{kms}^{-1}\right)\end{array}$ \\
\hline \multirow{3}{*}{$\mathrm{S} 1$} & Median & -1.78 & 3.96 & 6073.2 & $(8.1,-0.2,2.8)$ & $(44.8,-313.8,-42.7)$ & $(32.9,-322.6,-42.7)$ & 55872 & 2911 \\
\hline & MAD & 0.19 & 0.21 & 265.7 & $(0.4,0.9,1.1)$ & $(38.4,75.7,21.3)$ & $(25.8,65.4,21.3)$ & 22621 & 519 \\
\hline & Dispersion & 0.27 & 0.25 & 335.2 & $(0.8,1.2,1.9)$ & $(56.2,93.4,26.5)$ & $(39.2,86.5,26.5)$ & 26815 & 822 \\
\hline \multirow{3}{*}{$\mathrm{S} 2$} & Median & -1.91 & 4.00 & 5847.0 & $(8.7,0.3,0.8)$ & $(8.9,160.2,-249.9)$ & $(-1.2,164.9,-249.9)$ & 46048 & 2632 \\
\hline & MAD & 0.26 & 0.26 & 392.4 & $(0.3,0.6,1.5)$ & $(19.7,12.1,11.7)$ & $(17.1,10.2,11.7)$ & 2527 & 127 \\
\hline & Dispersion & 0.35 & 0.32 & 473.8 & $(0.6,1.0,2.0)$ & $(28.2,16.8,17.5)$ & $(35.6,15.5,17.5)$ & 4962 & 233 \\
\hline \multirow{3}{*}{$\mathrm{S} 3$} & Median & -1.34 & 4.05 & 6114.3 & $(8.6,0.5,3.5)$ & $(50.6,-245.5,206.7)$ & $(77.3,-257.0,206.7)$ & 59857 & 2901 \\
\hline & MAD & 0.08 & 0.20 & 196.8 & $(0.6,1.0,1.0)$ & $(67.3,30.5,19.0)$ & $(60.1,40.6,19.0)$ & 11230 & 448 \\
\hline & Dispersion & 0.13 & 0.30 & 334.2 & $(1.1,1.5,1.9)$ & $(80.1,58.7,22.4)$ & $(66.1,61.5,22.4)$ & 17604 & 792 \\
\hline \multirow{3}{*}{ S4 } & Median & -1.70 & 3.83 & 6144.1 & $(8.5,0.6,4.1)$ & $(4.0,-250.5,157.7)$ & $(14.7,-262.7,157.7)$ & 49617 & 2755 \\
\hline & MAD & 0.14 & 0.14 & 203.8 & $(0.5,1.2,1.8)$ & $(68.2,43.8,18.5)$ & $(41.3,38.3,18.5)$ & 11436 & 351 \\
\hline & Dispersion & 0.19 & 0.28 & 290.7 & $(1.0,, 1.7,3.6)$ & $(84.4,54.3,22.2)$ & $(61.6,56.2,22.2)$ & 14708 & 719 \\
\hline \multirow{3}{*}{$\mathrm{C} 1$} & Median & -2.11 & 3.85 & 6081.4 & $(8.7,-0.8,2.5)$ & $(32.0,32.3,271.6)$ & $(33.7,29.4,271.6)$ & 38235 & 2311 \\
\hline & MAD & 0.16 & 0.25 & 285 & $(0.4,1.0,1.1)$ & $(8.5,18.6,11.2)$ & $(10.7,20.4,11.2)$ & 2502 & 107 \\
\hline & Dispersion & 0.29 & 0.31 & 360 & $(0.9,1.4,1.6)$ & $(17.8,26.9,15.8)$ & $(18.2,28.3,15.8)$ & 4807 & 191 \\
\hline \multirow{3}{*}{$\mathrm{C} 2$} & Median & -1.39 & 4.05 & 5998.7 & $(8.9,-1.0,2.1)$ & $(-337.5,75.4,295.0)$ & $(-322.7,121.0,295.0)$ & 105895 & 3498 \\
\hline & MAD & 0.12 & 0.21 & 258.9 & $(0.6,0.3,0.3)$ & $(13.1,16.9,8.2)$ & $(15.3,27.8,8.2)$ & 3319 & 147 \\
\hline & Dispersion & 0.17 & 0.25 & 320.1 & $(0.85,0.5,0.6)$ & $(16.2,23.9,10.0)$ & $(18.6,32.3,10.0)$ & 6631 & 293 \\
\hline
\end{tabular}

Table 1. The median, median absolute deviation and dispersions in spectroscopic and kinematic properties of the six substructures. 

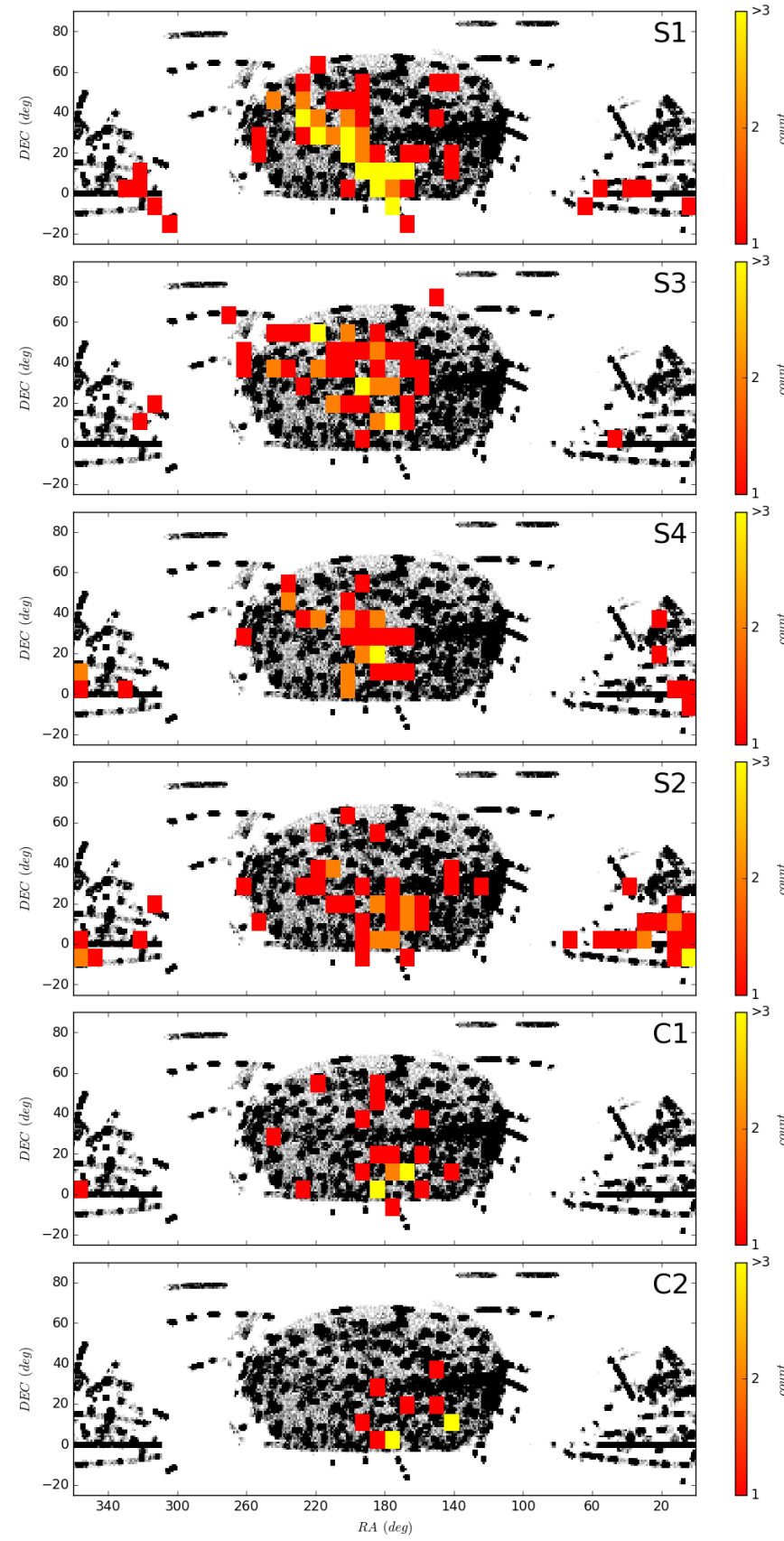

Figure 8. The location of the stars belong to the substructures are shown in the plane of right ascension versus declination. The pixel size is $8.5 \mathrm{deg}$ on each side.

\subsection{The Colder Substructures: S2, C1 and C2}

The top row of Fig. 6 shows the discovery panels for substructure S2 comprising 73 member stars, which has the characteristics of a halo stream. S2 corresponds to an obvious overdensity in the $\left(v_{\phi}, v_{z}\right)$ velocity distribution. It can be seen as an underhanging blob of stars in the lower rightmost panel of Fig. 2 at $\left(v_{\phi}, v_{z}\right) \simeq(160,-250)$ $\mathrm{kms}^{-1}$. The member stars also comprise a tight grouping in the $\left(v_{R}, v_{\phi}\right)$ and $\left(v_{R}, v_{z}\right)$ planes. The coldness of this substructure in velocity space is emphasised by the narrow velocity distributions. The median absolute deviation in $\left(v_{R}, v_{\phi}, v_{z}\right)$ are $(19.7,12.1,11.7) \mathrm{kms}^{-1}$, though these are of course averages over the spatial extent of the stream and so are not indicative of the velocity dispersion or the size of the progenitor.

The median values of the spatial coordinates are $(X, Y, Z)=$ $(8.7,0.3,0.8) \mathrm{kpc}$, so that this substructure is again close to the Sun. Nearby objects have the highest proper motions and stand out from the bulk of the stars in the catalogue, so it is not surprising that our detection is more sensitive to the substructures close to the Solar radius. As Fig. 6 shows, S2 is a stream plunging through the Galactic disk, moving on a nearly polar orbit. The fact that the stream is aligned along the velocity vectors of the stars, as is natural for a stream, adds confidence to our detection. The stars have a median metallicity $[\mathrm{Fe} / \mathrm{H}]$ of -1.91 and a median absolute deviation of 0.26. As is clear from Fig. 7, the metallicity distribution function of the substructure is poorer and narrower than the stellar halo as a whole.

In fact, $\mathrm{S} 2$ lies at a very similar location in velocity space as 4 stars belonging to the halo stream identified in Hipparcos data by Helmi et al. (1999, see especially the upper panels of their Fig. 2). Their stars are clumped in "integrals of motion space", while the two structures have no direct member stars in common, presumably due to the use of different dataset. The relationship of S2 with the stream of Helmi et al. (1999) will be discussed in detail elsewhere. As the associated substructure has been identified both in velocity space and in "integrals of motion space", it provides an interesting test case for assessing the advantages and disadvantages of each search arena and algorithm.

The middle and bottom rows of Fig. 6 show panels for the two clumps $\mathrm{C} 1$ and $\mathrm{C} 2$. These comprise 24 and 12 members respectively, and so are less substantial and extensive than S1-S4. Their velocity histograms are very narrow with the vertical velocity distribution being the coldest. The structures are tightly confined in space and in metallicity. The median metallicity $[\mathrm{Fe} / \mathrm{H}]$ of $\mathrm{C} 1$ is -2.11 , making it the most metal-poor of all our substructures, whilst $\mathrm{C} 2$ has a median metallicity of -1.39 (see Fig. 7).

\subsection{Distribution on the Sky}

The locations of the stars in right ascension and the declination for all the substructures are shown in Fig. 8. Notice that the substructure are difficult to discern, with the exceptions of S1 and S4. In general, the substructures are both nearby and extended, so their member stars are scattered across the sky. The stream S2 is hard to make out, as it is traversing the Galactic disk. Fig. 8 vindicates the power of kinematic searches, as the substructures would be nearly impossible to identify any other way. 

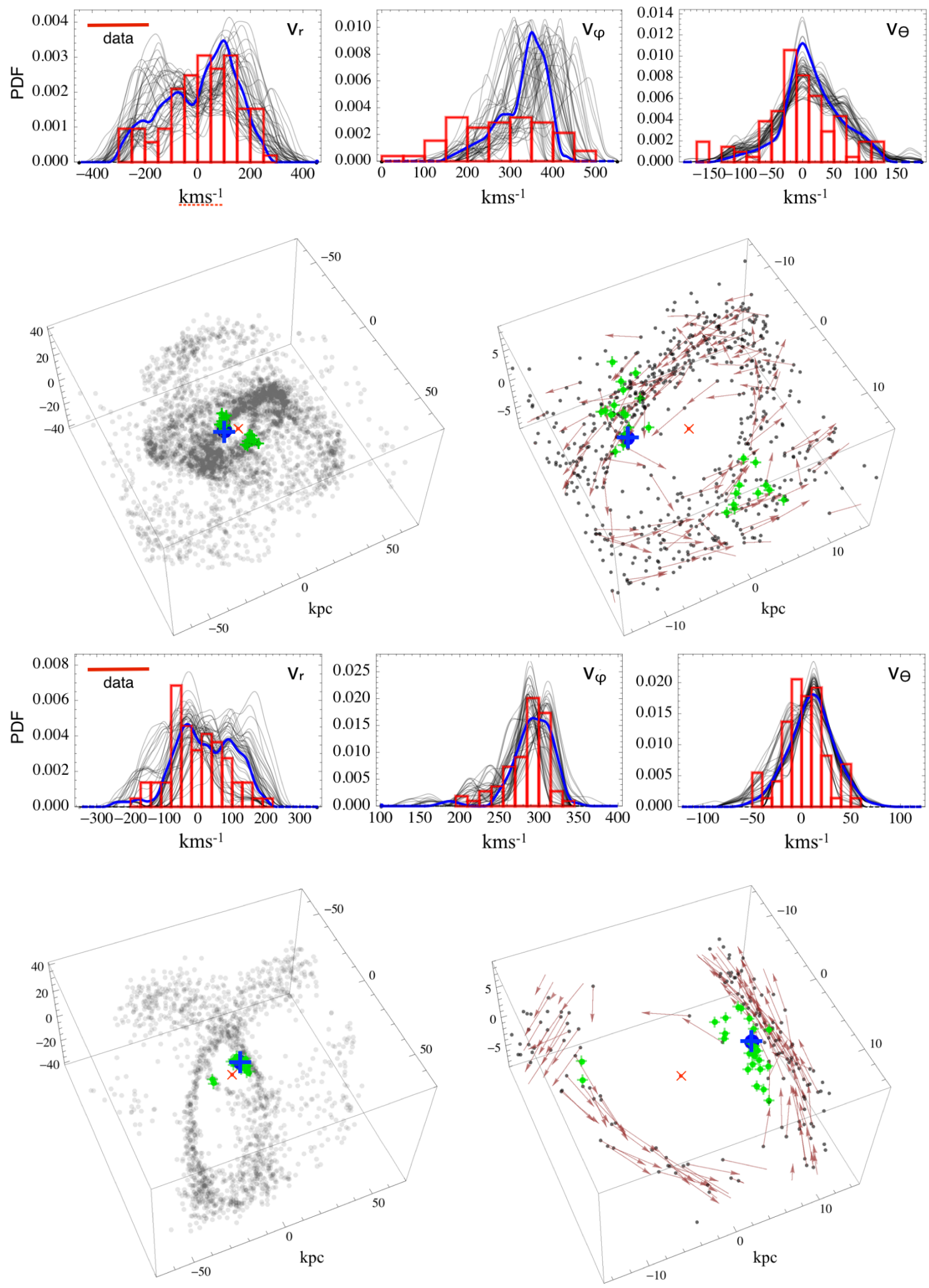

Figure 9. Matches to substructures S1 (upper two rows of panels) and S2 (lower two rows of panels) in the library of Amorisco (2017). For each substructure, the observed kinematics (red histograms) is compared with the chosen model in the upper trios of panels. There, thin grey lines illustrate the debris' kinematics corresponding to different viable positions of the Sun. The thick blue line identifies the best-fitting model, corresponding to the best Sun's position. The lower-left panels illustrate the three-dimensional structure of the simulated tidal debris. Grey points are model particles and the red X symbol identifies the Galactic centre. Green + symbols identify the Sun's positions corresponding to the kinematic distributions shown in the upper trio of panels. The best Sun's position is displayed with a large blue + symbol. The panel in the lower-right is a zoomed version that best shows the position of the simulated debris material with respect to the selected Sun's locations. Symbols are as in the lower-left panels. Additionally, a fraction of the model particles are accompanied by their velocity vectors, to illustrate the debris kinematics. 


\section{INTERPRETATION}

We use the library of accretion events created by Amorisco (2017) to find model analogues for the two largest substructures S1 and S2. The library uses minor merger N-body simulations to study how stellar material is deposited onto the host. Both host and infalling satellites are assumed to have spherical Navarro-Frenk-White profiles (Navarro et al. 1997), meaning that the Milky Way disk is not properly accounted for. The disk is not expected to cause substantial additional satellite disruption in satellites with total masses $M_{\text {sat }} \gtrsim 10^{9} M_{\odot}$ (D’Onghia et al. 2010; Garrison-Kimmel et al. 2017), but can alter the debris' orbits and it does increase the speed of the phase-mixing process (e.g., Helmi \& White 1999). This initial exploration neglects these effects. Specifically, we search for accretion events that result in substructures located close to the Solar radius and that provide reasonable matches to the velocity histograms. To do so, we use spherical polar coordinate, $\left(v_{r}, v_{\theta}, v_{\varphi}\right)$, defined by the mean angular momentum vector of the substructure itself. Therefore, $v_{\varphi}$ refers to rotation in the mean orbital plane, while the scatter in $v_{\theta}$ is a proxy of the structure's hotness. By automating this, we can explore a large number of models in the library with a variety of mass ratios $\left(-2 \lesssim \log M_{\text {sat }} / M_{\text {halo }} \lesssim-0.5\right)$, infall circularities $(0.2<j<0.8$, where $j$ is the ratio between angular momentum and the maximum angular momentum at the same energy) and infall times. For each model we look for matches by considering thousands of possible Sun's locations, together with slightly different mass and length normalisations. The former exploits the lack of a stellar disk in the models, the second explores the possible scatter in the values of the Galaxy's mass and concentration at the time of infall.

Despite the size of this library, there do not seem to be that many models that fit reasonably when actually compared with the histograms of S1. For a randomly picked Sun's location, most model structures feature the presence of multiple phase-space wraps, resulting in sharply double peaked $v_{r}$ distributions, with average close to zero or $\left\langle v_{r}\right\rangle \approx 0$. Instead, $\mathrm{S} 1$ is characterised by a broad and unimodal $v_{r}$ distribution, which contains $v_{r}=0$ and is not double peaked. To reproduce this, the Sun is required to lie very close to the pericenter of the debris' orbit. Among those models for which feasible locations for the Sun can be found, we illustrate one of the most successful in the upper panel of Fig. 9, in which green points display a selection of feasible Sun's locations. Each of these produce the velocity distributions plotted as thin black lines in the upper panels. The best choice for the Sun's location is shown in blue. The corresponding blue velocity distributions reproduce most of the features of the velocity histograms, though the match to the $v_{\varphi}$ distribution is poor. It corresponds to a virial mass ratio of $M_{\text {sat }} / M_{\text {halo }}=1: 20$ at infall, implying that the progenitor had a starting mass of $\approx 2 \times 10^{10} M_{\odot}$ at infall time $\approx 10 \mathrm{Gyr}$ ago, for a circularity at infall of $j=0.8$.

As shown in the lower plot of the upper panel of Fig. 9, S1 is identified as a stream in an advanced state of disintegration. The Sun appears to lie within the stream's wraps, while these are at pericenter. The quite advanced state of phase mixing and partial superposition of multiple stream wraps helps in reproducing the broad $v_{\varphi}$ distribution, although the model distributions still appear to remain somewhat tighter than suggested by the data. The stream does indeed pirouette around the Sun, but the substructure S1 is not a shell. In fact, its angular momentum is still high, as permitted by the low initial virial mass ratio. The $v_{r}$ distribution encompasses $v_{r}=0$, but it does so while the Sun is close to the stream's pericenter rather than to the apocenter as in a more classical shell. The fact that one of the velocity distributions is poorly fit does mean that our conclusions regarding the properties of the progenitor of $\mathrm{S} 1$ are preliminary. It may be that we have a restricted view of S1 owing to the incompleteness of our sample, though integration of the orbits of the stars does not reveal a connection to other known substructures.

The $v_{r}$ histogram of the substructure $\mathrm{S} 2$ has a similar distinguishing property, implying that the Sun's preferred position is again very close to pericentre. The main difference is that the dispersions are smaller, which drives the model to lower mass ratios, and therefore to less phase-mixed morphologies. The lower panel of Fig. 9 illustrates a model that provides a good match: it has a virial mass ratio of $1: 100$ at infall, implying that the progenitor had a starting mass of $\approx 5 \times 10^{9} M_{\odot}$ at infall time $\approx 11 \mathrm{Gyr}$ ago, and an initial circularity of $j=0.5$. However, a number of models that are close in parameter space can also roughly reproduce the features of the substructure. For example, one can trade a slightly higher initial mass ratio $(\approx 1: 50)$ for a somewhat later infall time $(\approx 8 \mathrm{Gyr})$ or a marginally higher angular momentum. These coupled changes can compensate each other, without affecting much the degree of the stream's phase mixing, and therefore its kinematic properties.

As shown by the analysis above, a significant variety of models have a pericentric distance that is comparable with the Sun's radius. These are models with comparatively old progenitors, which helps them to fall deeper in the Milky Way halo, but not overly massive, which would instead cause excessive dynamical friction and phase mixing. Despite the limits of the models we adopted, it is clear that the progenitors of both $\mathrm{S} 1$ and $\mathrm{S} 2$ belong to this class.

The inferred total masses of $\mathrm{S} 1\left(\approx 10^{10} M_{\odot}\right)$ and $\mathrm{S} 2(\approx 5 \times$ $10^{9} M_{\odot}$ are about a factor of 10 smaller than the total mass of the Large Magellanic Cloud. According to the abundance matching of Garrison-Kimmel et al. (2014), these correspond to stellar masses between $10^{6}$ and $10^{7} M_{\odot}$, and so are comparable to present-day objects like the Fornax dwarf spheroidal. The stellar masses inferred for S1 and S2 through the mass-metallicity relation of Kirby et al. (2013) are $10^{5.7} M_{\odot}$ and $10^{5.3} M_{\odot}$, which are somewhat lower by factors of 5 to 15 . However, this does not take into account the redshift evolution of the mass-metallicity relation (see e.g., Ma et al. 2016), which though uncertain may remove these inconsistencies entirely. In addition, there is substantial scatter in both abundance matching, the mass-metallicity relation and the data of Kirby et al. (2013). Hence, metallicity and kinematics appear to be painting a broadly consistent picture.

Nonetheless, there are some clear shortcomings to our methodology. First, we did not use the footprint of the SDSS-Gaia survey and so this weakens our claim to a proper comparison with the data. Secondly, the proper motion errors are not known on a star by star basis, though on average they are reckoned to be $\sim 2$ mas $\mathrm{yr}^{-1}$. The effect of the proper motion errors is to broaden the distributions in the angular coordinates especially and this may partially explain our failure to reproduce the broadness of the $v_{\varphi}$ distribution for S1. Finally, the underlying galaxy models used to generate our substructure library are spherical and so somewhat idealised.

\section{CONCLUSIONS}

We have devised a method to search algorithmically for substructure. We model the distribution of the underlying smooth component as a Gaussian Mixture model. We use this to identify enhancements against the background, by comparing the local den- 
sity around any star with the prediction from the Gaussian Mixture model and thence computing the significance. Stars with significance greater than 4 are then grouped by a Friends-of-Friends algorithm to give substructures. In our application, the underlying smooth component is the velocity distribution of the stellar halo, and we were seeking kinematically coherent substructures that are the residue of long-disrupted dwarf galaxies.

Our method has a number of advantages. First, the entire algorithm is very fast. For the halo samples studied here (63742 stars), substructures can be identified and their significance computed in $\sim 100$ s. It is estimated that there will be $2 \times 10^{7}$ halo stars in Gaia Data Release 2 (Robin et al. 2012), so the algorithm remains competitive and feasible in the face of the much larger datasets expected shortly. Secondly, the algorithm is easily adapted to different search spaces. Here, we chose to search only in velocity space and use any metallicity data as confirmation. However, it would have been easy to add extra dimensions in chemistry (such as metallicity or abundances) and search in a chemo-dynamical space. Alternatively, we could have applied the algorithm in action or 'integral of motion' space.

We implemented the new algorithm on a sample of stars extracted from the SDSS-Gaia catalogue (see e.g. Deason et al. 2017). This uses Sloan Digital Sky Survey (SDSS) photometry as the first epoch for sources in Gaia DR1. When cross-matched with available spectroscopic surveys, such as RAVE, APOGEE or LAMOST, we obtain the line-of-sight velocities and metallicity. By photometrically selecting main sequence turn-off stars or BHB stars, for which distance estimators are available, we construct a sample of 245316 stars with full phase space coordinates. The velocity distributions show a strong trend of increasing substructure with diminishing metallicity. The most metal-poor stars $([\mathrm{Fe} / \mathrm{H}]<-1.65)$ exhibit abundant substructure in their velocity distributions. Some of the substructures are visible by eye.

Our new algorithm enabled us to identify six new substructures in the local stellar halo. The most substantial (S1) is a stream in an advanced state of disruption just beyond the Solar radius. The Sun is located close to the pericentre of multiple wraps, giving rise to a broad distribution in two of the velocity components. This is the relic of an old accretion event in which a satellite was engulfed on a retrograde orbit. Modelling suggests that the progenitor was relatively massive at $\approx 2 \times 10^{10} M_{\odot}$ at infall time $\approx 10 \mathrm{Gyr}$ ago. The next most substantial (S2) is a stream, though it is more intact. Again, it is located close to the Solar radius, but is plunging through the Galactic disk. It has characteristic stream kinematics, with the velocity vectors of the stars aligned with the elongation of the substructure. The cold velocity distributions suggest that the progenitor was less massive - at most perhaps $\approx 5 \times 10^{9} M_{\odot}$ at infall time $\approx 11 \mathrm{Gyr}$ ago. The stars belonging to these substructures are clustered not just kinematically but also chemically, which adds confidence to the detections. Abundance matching suggests that both $\mathrm{S} 1$ and $\mathrm{S} 2$ correspond to galaxies with stellar masses between $10^{6}$ and $10^{7} M_{\odot}$. This is comparable to the largest dwarf spheroidal galaxies surrounding the Milky Way today. The metallicities of S1 and S2 $([\mathrm{Fe} / \mathrm{H}] \approx-1.78$ and -1.91 respectively) are consistent with stellar masses of $\sim 10^{5.5}$ through the mass-metallicity relation (Kirby et al. 2013). Although such masses are slightly lower than our modelling suggests, it must be remembered that there is considerable scatter in both the abundance matching and the redshift dependence of the mass-metallicity relations.

We identified four further pieces of substructure; namely, two moving groups or clumps ( $\mathrm{C} 1$ and $\mathrm{C} 2)$ and two substructures (S3 and S4). The latter two share some similarities with $\mathrm{S} 1$ and are also probably streams in the later stages of disintegration. As all our substructures are nearby, the member stars are candidates for high resolution spectroscopic follow-up to provide abundances and ages. The larger substructures probably extend beyond the volume accessible in SDSS-Gaia, and it would be valuable to trace their full extent.

The overall aim of activity in this field is to provide an assessment of the fractional mass in substructure as a function of Galactic position and metallicity. Nevertheless, in Rutherford's words, the 'Stamp Collecting' is still insightful. It is useful to understand the largest substructures in the nearby halo and the the nature of the accretion events that gave rise to them. Our matches with the remnants of accretion events in libraries of numerically constructed stellar halos have provided insights, but they are not perfect - for example, we failed to reproduce the full broadness of the azimuthal velocity distribution in the case of S1. In fact, it was difficult to find perfect matches, even though our task was eased by the absence of a Galactic disk in the library of Amorisco (2017). This suggests that the problem of matching substructures in Gaia DR2 to accreted subhalos in simulations may be challenging.

\section{ACKNOWLEDGEMENTS}

GCM thanks Boustany Foundation, Cambridge Commonwealth, European \& International Trust and Isaac Newton Studentship for their support on his work. NWE thanks the Max Planck Institute for Astrophysics for hospitality during a working visit. We are grateful to the anonymous referee helped us improve the presentation and to Amina Helmi for providing data on halo stream stars. The research leading to these results has received partial support from the European Research Council under the European Union's Seventh Framework Programme (FP/2007-2013) / ERC Grant Agreement no. 308024.

\section{REFERENCES}

Amorisco, N. C. 2015, MNRAS, 450, 575

Amorisco, N. C. 2017, MNRAS, 464, 2882

Anders, F., Chiappini, C., Santiago, B. X., et al. 2014, A\&A, 564, A115

Belokurov, V., Zucker, D. B., Evans, N. W., et al. 2006, ApJ, 642, L137

Belokurov, V., Evans, N. W., Bell, E. F., et al. 2007, ApJ, 657, L89

Casey, A. R., Hawkins, K., Hogg, D. W., et al. 2017, ApJ, 840, 59

de Boer, T. J. L., Belokurov, V., \& Koposov, S. E. 2018, MNRAS, 473, 647

Deason, A. J., Belokurov, V., Evans, N. W., 2011, MNRAS, 416, 2903

Deason, A. J., Belokurov, V., Koposov, S. E., et al. 2017, arXiv:1703.09230

D’Onghia, E., Springel, V., Hernquist, L., \& Keres, D. 2010, ApJ, 709, 1138

Gaia Collaboration, Prusti, T., de Bruijne, J. H. J., et al. 2016a, A\&A, 595, A1

Gaia Collaboration, Brown, A. G. A., Vallenari, A., et al. 2016b, A\&A, 595, A2

Garrison-Kimmel, S., Boylan-Kolchin, M., Bullock, J. S., \& Lee, K. 2014, MNRAS, 438, 2578

Garrison-Kimmel, S., Wetzel, A., Bullock, J. S., et al. 2017, MNRAS, 471, 1709

Grillmair, C. J. 2009, ApJ, 693, 1118

Helmi, A., White, S. D. M., de Zeeuw, P. T., \& Zhao, H. 1999, Nature, 402, 53

Helmi, A., Veljanoski, J., Breddels, M. A., Tian, H., \& Sales, L. V. 2017, A\&A, 598, A58

Helmi, A., \& White, S. D. M. 1999, MNRAS, 307, 495

Hendel, D., \& Johnston, K. V. 2015, MNRAS, 454, 2472

Hernquist, L., \& Quinn, P. J. 1987, ApJ, 312, 1

Ivezic, Z, et al. 2008, ApJ, 684, 287 
Kirby, E. N., Boylan-Kolchin, M., Cohen, J. G., et al. 2013, ApJ, 770, 16

Kunder, A., Kordopatis, G., Steinmetz, M., et al. 2017, AJ, 153, 75

Luo, A.-L., Zhao, Y.-H., Zhao, G., et al. 2015, Research in Astronomy and Astrophysics, 15, 1095

Ma, X., Hopkins, P. F., Faucher-Giguère, C.-A., et al. 2016, MNRAS, 456, 2140

Myeong, G. C., Evans, N. W., Belokurov, V., Koposov, S. E., \& Sanders, J. L. 2017, MNRAS, 469, L78

Navarro, J. F., Frenk, C. S., \& White, S. D. M. 1997, ApJ, 490, 493

Newberg H. J., Carlin J. L. 2015, Tidal Streams in the Local Group and Beyond, Springer, New York

Pedregosa, F., Varoquaux, G., Gramfort, A., et al. 2011, Journal of Machine Learning Research, 12, 2825

Pop, A.-R., Pillepich, A., Amorisco, N. C., \& Hernquist, L. 2017, arXiv: 1706.06102

Robin, A. C., Luri, X., Reylé, C., et al. 2012, A\&A, 543, A100

Rocha-Pinto, H. J., Majewski, S. R., Skrutskie, M. F., Crane, J. D., \& Patterson, R. J. 2004, ApJ, 615, 732

Smith, M. C., Evans, N. W., Belokurov, V., et al. 2009, MNRAS, 399, 1223

Williams, A. A., Belokurov, V., Casey, A. R., \& Evans, N. W. 2017, MNRAS, 468, 2359 